\title{
An Alternative Proof of an Extension Theorem of T. Ohsawa
}

\author{
Klas Diederich \& Gregor Herbort
}

\section{Introduction}

In [DHOh; OhT; Oh2; Oh3], extension theorems for weighted square-integrable holomorphic functions that are defined on intersections of lower-dimensional affine subspaces with a pseudoconvex domain $D$ were proved on the basis of $L^{2}$-estimates for the $\bar{\partial}$-operator. (See also [Oh2; De; Bou; Mv] for generalizations to holomorphic differential forms with values in certain vector bundles.) They have proved to be useful in many applications, among them the behavior of the Bergman kernel [DH2; McN; JP] and the construction of integral kernels for the $\bar{\partial}$-equation, [BonD].

It is therefore of interest to have proofs for such extension results that are as elementary as possible. For the theorem of Ohsawa and Takegoshi (see [OhT]), such new proofs have been given, for instance, in [Bs; $\mathrm{McN}$; Siu] and also by T. Ohsawa himself (oral communication). Our goal here is to give also an elementary proof for the refined extension theorem of Ohsawa [Oh3] that allows so-called negligible weights in the extension. Our proof will be free of tools from Kähler geometry.

Let us first clarify some notations and state the theorem. Let $D \subset \mathbb{C}^{n}$ be an arbitrary pseudoconvex domain in $\mathbb{C}^{n}$. For a plurisubharmonic function $\psi$ on $D$, we denote by $H^{2}(D, \psi)$ the Hilbert space of holomorphic functions in $L^{2}(D, \psi)$. We also fix an affine linear subspace $H \subset \mathbb{C}^{n}$ of codimension $k$ for which $D^{\prime}=$ $D \cap H \neq \emptyset$. Then the extension theorem of [Oh3] can be stated in the following form.

0.1. Theorem. Assume that there exists on $D$ a plurisubharmonic function $V$ such that

$$
C_{V}:=\sup _{D}(V+2 k \log \operatorname{dist}(\cdot, H))<\infty .
$$

Then there exists a continuous linear extension operator $E_{\psi}^{V}: H^{2}\left(D^{\prime}, \psi+V\right) \rightarrow$ $H^{2}(D, \psi)$ whose operator norm is bounded by

$$
\left\|E_{\psi}^{V}\right\|^{2} \leq C_{n} e^{C_{V}} .
$$

The constant $C_{n}$ depends only on the dimension and not on the choice of $\psi$ and $D$.

Received September 10, 1998. Revision received February 9, 1999.

Michigan Math. J. 46 (1999). 
In Section 1 we will reduce the proof to a simpler situation. Section 2 contains the basic estimate that we use. The proof of Theorem 0.1 will be given in Section 3 , and Section 4 contains our application to the Bergman kernel.

\section{Reduction Steps}

Let $D, D^{\prime}, \psi$, and $V$ be as in Theorem 0.1. At first we show that it is enough to prove:

Any $f \in H^{2}\left(D^{\prime}, \psi+V\right)$ admits a holomorphic extension $\tilde{f} \in H^{2}(D, \psi)$ such that

$$
\|\tilde{f}\|_{\psi}^{2} \leq C_{n} e^{C_{V}}\|f\|_{D^{\prime}, \psi+V}^{2} .
$$

Namely, suppose that we have shown this. Let $h^{2}(D, \psi)$ denote the (closed) subspace of all functions $g \in H^{2}(D, \psi)$ such that $g \mid D^{\prime}=0$, and denote by $\pi^{\prime}: H^{2}(D, \psi) \rightarrow h^{2}(D, \psi)$ the orthogonal projection. Then, for a function $f \in$ $H^{2}\left(D^{\prime}, \psi+V\right)$, we choose an extension $\tilde{f} \in H^{2}(D, \psi)$ and put

$$
E_{\psi}^{V}(f):=\tilde{f}-\pi^{\prime}(\tilde{f}) .
$$

It is easy to check that this definition is independent of the choice of the extension $\tilde{f}$ and hence is consistent. Also, it is elementary to show that $E_{\psi}^{V}$ defines the desired extension operator.

For the proof of (1), the following further reductions are possible. Let $\Phi: D \rightarrow$ $\mathbb{R}^{+}$be a strongly plurisubharmonic exhaustion function and $T \subset \mathbb{R}^{+}$an unbounded set such that $D_{t}:=\{\Phi<t\}$ is strictly pseudoconvex with a smooth boundary for all $t \in T$. Let $D_{t}^{\prime}=D_{t} \cap H$.

A routine argument based upon the Alaoglu-Bourbaki theorem on weak- $\star-$ compactness of the unit ball in a normed space then justifies that it suffices to show:

For each $t \in T$ and $f \in H^{2}\left(D_{t}^{\prime}, \psi+V\right)$, there is an extension $\tilde{f}_{t} \in$ $H^{2}\left(D_{t}, \psi\right)$ for $f$ satisfying

$$
\left\|\tilde{f}_{t}\right\|_{D_{t}, \psi}^{2} \leq C_{n} e^{C_{V}}\|f\|_{D^{\prime}, \psi+V}^{2}
$$

with a constant $C_{n}$ independent of $\psi$ and $t$.

Finally it obviously suffices to prove (2) under the additional assumption that $\psi$ and $V$ are smooth. Namely, on $D_{t}$ one can choose decreasing sequences $\left(\psi_{s}\right)_{s}$ and $\left(V_{s}\right)_{s}$ of smooth plurisubharmonic functions that converge to $\psi$ and $V$, respectively. Then (2) applies with $\psi_{s}$ and $V_{s}$ instead of $\psi$ and $V$. Hence the smoothness assumption on $\psi$ and $V$ can be removed by applying the Alaoglu-Bourbaki theorem once more.

\section{A Basic Estimate for the $\bar{\partial}$ Operator}

Let $\Omega$ denote a smooth bounded domain in $\mathbb{C}^{n}$ with a defining function $r$ that is normalized in such a way that $|\nabla r|=1$ on $\partial \Omega$. Let $\varphi$ be a $C^{2}$-smooth function on 
$\bar{\Omega}$. Let $q \in\{0, \ldots, n-1\}$. The standard $\bar{\partial}$-operator on $L_{(0, q)}^{2}(\Omega, \varphi)$ has a closure, also denoted by $\bar{\partial}$. By $\bar{\partial}_{\varphi}^{*}: L_{(0, q+1)}^{2}(\Omega, \varphi) \rightarrow L_{(0, q)}^{2}(\Omega, \varphi)$ we denote the closure of the formal adjoint of $\bar{\partial}$. The space

$$
\mathcal{F}_{q}(\Omega, \varphi):=C_{(0, q+1)}^{1}(\bar{\Omega}) \cap \operatorname{dom}\left(\bar{\partial}_{\varphi}^{*}\right)
$$

consists of all $(0, q+1)$-forms $u \in C_{(0, q+1)}^{1}(\bar{\Omega})$ satisfying the Neumann condition

$$
u\lrcorner \partial r=0 \quad \text { on } \partial \Omega .
$$

This space is known to be dense in $L_{(0, q+1)}^{2}(\Omega, \varphi) \cap \operatorname{dom}\left(\bar{\partial}_{\varphi}^{*}\right)$ with respect to the graph norm $u \mapsto\|u\|_{\varphi}+\|\bar{\partial} u\|_{\varphi}+\left\|\bar{\partial}_{\varphi}^{*} u\right\|_{\varphi}$ (see [Hör, p. 100]).

For a function $f \in C^{2}(\bar{\Omega})$ and a $(0,1)$-form $u=\sum_{j=1}^{n} u_{j} d \bar{z}_{j}$, we write for short

$$
\mathcal{L}_{f}(z ; u):=\sum_{j, k=1}^{n} \frac{\partial^{2} f}{\partial z_{j} \partial \bar{z}_{k}}(z) u_{j}(z) \overline{u_{k}}(z)
$$

2.1. Lemma (The a priori formula for $\bar{\partial}$ ). Let $\Omega$ and $\varphi$ be as before and let $\eta \in$ $C^{2}(\bar{\Omega})$ be a positive function. Then for $u=u_{1} d \bar{z}_{1}+\cdots+u_{n} d \bar{z}_{n} \in \mathcal{F}_{1}(\Omega, \varphi)$ we have

(a)

$$
\begin{aligned}
\|\sqrt{\eta} \bar{\partial} u\|_{\varphi}^{2}+\left\|\sqrt{\eta} \bar{\partial}_{\varphi}^{*} u\right\|_{\varphi}^{2} \\
\left.=\int_{D_{t}}\left(\eta \mathcal{L}_{\varphi}-\mathcal{L}_{\eta}\right)(z ; u) e^{-\varphi} d \lambda(z)+2 \operatorname{Re}(u\lrcorner \partial \eta, \bar{\partial}_{\varphi}^{*} u\right)_{\varphi} \\
\quad+\sum_{i, j=1}^{n} \int_{\Omega} \eta\left|\frac{\partial u_{i}}{\partial \bar{z}_{j}}\right|^{2} e^{-\varphi} d \lambda+\int_{\partial \Omega} \eta \mathcal{L}_{r}(\zeta ; u) e^{-\varphi} d \sigma(\zeta) ;
\end{aligned}
$$

(b) in particular, if $\Omega$ is pseudoconvex then

$$
\begin{aligned}
& \|\sqrt{\eta} \bar{\partial} u\|_{\varphi}^{2}+\left\|\sqrt{\eta} \bar{\partial}_{\varphi}^{*} u\right\|_{\varphi}^{2} \\
& \left.\quad \geq \int_{\Omega}\left(\eta \mathcal{L}_{\varphi}-\mathcal{L}_{\eta}\right)(z ; u) e^{-\varphi} d \lambda(z)+2 \operatorname{Re}(u\lrcorner \partial \eta, \bar{\partial}_{\varphi}^{*} u\right)_{\varphi} .
\end{aligned}
$$

Here $d \lambda$ denotes the Lebesgue measure and $d \sigma$ the area measure on $\partial \Omega$.

Proof. The first formula is stated in [BoS]. For the reader's convenience we include a proof here. It uses the same technique (based upon integration by parts) as applied by Hörmander [Hör]. Similar computations have been carried out in [McN; Bs; Siu]. First we recall the integral formula of Gauss:

$$
\int_{\Omega} \frac{\partial f}{\partial z_{j}} \bar{g} d \lambda=-\int_{\Omega} f \frac{\partial \bar{g}}{\partial z_{j}} d \lambda+\int_{\partial \Omega} \frac{\partial r}{\partial z_{j}} f \bar{g} d \sigma
$$

for functions $f, g \in C^{1}(\bar{\Omega})$. For $1 \leq k \leq n$, let $\delta_{k}$ denote the operator

$$
\delta_{k} h=e^{\varphi} \frac{\partial\left(e^{-\varphi} h\right)}{\partial z_{k}} \quad \text { for } h \in C^{1}(\bar{\Omega}) .
$$


With this notation, for $u=u_{1} d \bar{z}_{1}+\cdots+u_{n} d \bar{z}_{n} \in \mathcal{F}_{1}(\Omega, \varphi)$ we can write

$$
\bar{\partial}_{\varphi}^{*} u=-\sum_{j=1}^{n} \delta_{j} u_{j} \text {. }
$$

We start by computing

$$
\left\|\sqrt{\eta} \bar{\partial}_{\varphi}^{*} u\right\|_{\varphi}^{2}=\sum_{j, k=1}^{n} \int_{\Omega} \eta \delta_{j} u_{j} \overline{\delta_{k} u_{k}} e^{-\varphi} d \lambda,
$$

using integration by parts. From formula (6) for $f=e^{-\varphi} u_{j}$ and $g=\eta \delta_{k}\left(u_{k}\right)$, we obtain

$$
\begin{aligned}
\int_{\Omega} \eta \delta_{j}\left(u_{j}\right) \overline{\delta_{k}\left(u_{k}\right)} e^{-\varphi} d \lambda= & \int_{\Omega} \eta \frac{\partial\left(e^{-\varphi} u_{j}\right)}{\partial z_{j}} \overline{\delta_{k}\left(u_{k}\right)} d \lambda \\
= & -\int_{\Omega} e^{-\varphi} u_{j} \frac{\partial\left(\eta \overline{\delta_{k}\left(u_{k}\right)}\right)}{\partial z_{j}} d \lambda+\int_{\partial \Omega} \frac{\partial r}{\partial z_{j}} u_{j} \overline{\delta_{k} u_{k}} \eta e^{-\varphi} d \sigma \\
= & -\int_{\Omega} e^{-\varphi} u_{j} \frac{\partial \eta}{\partial z_{j}} \overline{\delta_{k}\left(u_{k}\right)} d \lambda-\int_{\Omega} e^{-\varphi} u_{j} \eta \frac{\partial\left(\overline{\delta_{k}\left(u_{k}\right)}\right)}{\partial z_{j}} d \lambda \\
& +\mathcal{I}_{1}(j, k),
\end{aligned}
$$

where

$$
\mathcal{I}_{1}(j, k):=\int_{\partial \Omega} \frac{\partial r}{\partial z_{j}} u_{j} \overline{\delta_{k} u_{k}} \eta e^{-\varphi} d \sigma .
$$

Now one has a commutator relation for the $\delta_{k}$, namely,

$$
\left[\delta_{k}, \frac{\partial}{\partial \bar{z}_{j}}\right]=\frac{\partial^{2} \varphi}{\partial z_{k} \partial \bar{z}_{j}} .
$$

Substituting this into the second member on the right-hand side of (8), we have

$$
\begin{aligned}
-\int_{\Omega} e^{-\varphi} u_{j} \eta \frac{\partial\left(\overline{\delta_{k}\left(u_{k}\right)}\right)}{\partial z_{j}} d \lambda & =-\int_{\Omega} e^{-\varphi} u_{j} \eta \frac{\overline{\partial\left(\delta_{k}\left(u_{k}\right)\right)}}{\partial \bar{z}_{j}} d \lambda \\
& =\int_{\Omega} e^{-\varphi} u_{j} \eta \overline{\left(\frac{\partial^{2} \varphi}{\partial z_{k} \partial \bar{z}_{j}}-\delta_{k} \frac{\partial}{\partial \bar{z}_{j}}\right) u_{k}} d \lambda \\
& =\int_{\Omega} e^{-\varphi} \eta \frac{\partial^{2} \varphi}{\partial z_{j} \partial \bar{z}_{k}} u_{j} \overline{u_{k}} d \lambda-\overline{\int_{\Omega} e^{-\varphi} \eta \overline{u_{j}} \delta_{k} \frac{\partial u_{k}}{\partial \bar{z}_{j}} d \lambda .}
\end{aligned}
$$

The second integral on the right side is again transformed by Gauss' formula:

$$
\begin{aligned}
-\int_{\Omega} e^{-\varphi} \eta \overline{u_{j}} \delta_{k}\left(\frac{\partial u_{k}}{\partial \bar{z}_{j}}\right) d \lambda= & -\int_{\Omega} \eta \overline{u_{j}} \frac{\partial}{\partial z_{k}}\left(e^{-\varphi} \frac{\partial}{\partial \bar{z}_{j}} u_{k}\right) d \lambda \\
= & \int_{\Omega} e^{-\varphi} \eta \frac{\partial \overline{u_{j}}}{\partial z_{k}} \frac{\partial u_{k}}{\partial \bar{z}_{j}} d \lambda \\
& +\int_{\Omega} \frac{\partial \eta}{\partial z_{k}} \bar{u}_{j} \frac{\partial u_{k}}{\partial \bar{z}_{j}} e^{-\varphi} d \lambda-\mathcal{I}_{2}^{\prime}(j, k),
\end{aligned}
$$


where

$$
\mathcal{I}_{2}^{\prime}(j, k)=\int_{\partial \Omega} \eta \frac{\partial r}{\partial z_{k}} \overline{u_{j}} \frac{\partial u_{k}}{\partial \bar{z}_{j}} e^{-\varphi} d \sigma .
$$

The complex conjugate of this is

$-\overline{\int_{\Omega} e^{-\varphi} \eta \overline{u_{j}} \delta_{k} \frac{\partial u_{k}}{\partial \bar{z}_{j}} d \lambda}=\int_{\Omega} e^{-\varphi} \eta \frac{\partial u_{j}}{\partial \bar{z}_{k}} \frac{\overline{\partial u_{k}}}{\partial \bar{z}_{j}} d \lambda+\int_{\Omega} \frac{\partial \eta}{\partial \bar{z}_{k}} u_{j} \frac{\overline{\partial u_{k}}}{\partial \bar{z}_{j}} e^{-\varphi} d \lambda-\mathcal{I}_{2}(j, k)$,

where

$$
\mathcal{I}_{2}(j, k):=\int_{\partial \Omega} \eta \frac{\partial r}{\partial \bar{z}_{k}} u_{j} \frac{\partial \overline{u_{k}}}{\partial z_{j}} e^{-\varphi} d \sigma .
$$

If we substitute this into the computations carried out so far, we obtain

$$
\begin{aligned}
\int_{\Omega} \eta \delta_{j}\left(u_{j}\right) \overline{\delta_{k}\left(u_{k}\right)} e^{-\varphi} d \lambda= & -\int_{\Omega} u_{j} \frac{\partial \eta}{\partial z_{j}} \overline{\delta_{k} u_{k}} e^{-\varphi} d \lambda+\int_{\Omega} \frac{\partial \eta}{\partial \bar{z}_{k}} u_{j} \frac{\overline{\partial u_{k}}}{\partial \bar{z}_{j}} e^{-\varphi} d \lambda \\
& +\int_{\Omega} \eta \frac{\partial^{2} \varphi}{\partial z_{j} \partial \bar{z}_{k}} u_{j} \overline{u_{k}} e^{-\varphi} d \lambda+\int_{\Omega} \eta \frac{\partial u_{j}}{\partial \bar{z}_{k}} \overline{\frac{\partial u_{k}}{\partial \bar{z}_{j}}} e^{-\varphi} d \lambda \\
& +\mathcal{I}_{1}(j, k)-\mathcal{I}_{2}(j, k) .
\end{aligned}
$$

Next we want to sum this over all indices $j, k \in\{1, \ldots, n\}$. The Neumann condition (3) at the level of $(0,1)$-forms reduces to

$$
\sum_{j=1}^{n} u_{j} \frac{\partial r}{\partial z_{j}}=0 \quad \text { on } \partial \Omega
$$

in particular, $\sum_{j=1}^{n} \mathcal{I}_{1}(j, k)=0$ for all $k=1, \ldots, n$. Hence we obtain

$$
\begin{aligned}
\left\|\sqrt{\eta} \bar{\partial}_{\varphi}^{*} u\right\|_{\varphi}^{2}= & -\int_{\Omega} \sum_{j, k} u_{j} \frac{\partial \eta}{\partial z_{j}} \overline{\delta_{k} u_{k}} e^{-\varphi} d \lambda+\int_{\Omega} \sum_{j, k} \frac{\partial \eta}{\partial \bar{z}_{k}} u_{j} \frac{\overline{\partial u_{k}}}{\partial \bar{z}_{j}} e^{-\varphi} d \lambda \\
& +\int_{\Omega} \eta \sum_{j, k} \frac{\partial^{2} \varphi}{\partial z_{j} \partial \bar{z}_{k}} u_{j} \overline{u_{k}} e^{-\varphi} d \lambda+\int_{\Omega} \sum_{j, k} \eta \frac{\partial u_{j}}{\partial \bar{z}_{k}} \frac{\partial u_{k}}{\partial \bar{z}_{j}} e^{-\varphi} d \lambda \\
& -\sum_{j, k} \mathcal{I}_{2}(j, k) .
\end{aligned}
$$

We now observe (see [Hör, p. 102]) that

$$
\|\sqrt{\eta} \bar{\partial} u\|_{\varphi}^{2}=\int_{\Omega} \eta\left(\sum_{j, k=1}^{n}\left|\frac{\partial u_{k}}{\partial \bar{z}_{j}}\right|^{2}-\sum_{j, k} \frac{\partial u_{j}}{\partial \bar{z}_{k}} \frac{\overline{\partial u_{k}}}{\partial \bar{z}_{j}}\right) e^{-\varphi} d \lambda .
$$

This is substituted into (11) to yield

$$
\begin{aligned}
\|\sqrt{\eta} \bar{\partial} u\|_{\varphi}^{2}+\left\|\sqrt{\eta} \bar{\partial}_{\varphi}^{*} u\right\|_{\varphi}^{2}= & \int_{\Omega} \eta \sum_{j, k=1}^{n}\left|\frac{\partial u_{k}}{\partial \bar{z}_{j}}\right|^{2} e^{-\varphi} d \lambda+T_{2}+T_{3} \\
& +\int_{\Omega} \eta \sum_{j, k=1}^{n} \frac{\partial^{2} \varphi}{\partial z_{j} \partial \bar{z}_{k}} u_{j} \overline{u_{k}} e^{-\varphi} d \lambda-\sum_{j, k} \mathcal{I}_{2}(j, k),
\end{aligned}
$$


where

$$
T_{2}:=-\int_{\Omega} \sum_{j, k} u_{j} \frac{\partial \eta}{\partial z_{j}} \overline{\delta_{k} u_{k}} e^{-\varphi} d \lambda \quad \text { and } \quad T_{3}:=\int_{\Omega} \sum_{j, k} \frac{\partial \eta}{\partial \bar{z}_{k}} u_{j} \frac{\overline{\partial u_{k}}}{\partial \bar{z}_{j}} e^{-\varphi} d \lambda .
$$

Finally, the proof of (a) will be complete if we show that the sum of the second and third terms on the right $\left(T_{2}\right.$ and $\left.T_{3}\right)$ is equal to

$$
\left.-\int_{\Omega} \sum_{j, k=1}^{n} \frac{\partial^{2} \eta}{\partial z_{j} \partial \bar{z}_{k}} u_{j} \overline{u_{k}} e^{-\varphi} d \lambda+2 \operatorname{Re}(u\lrcorner \partial \eta, \bar{\partial}_{\varphi}^{*} u\right)_{\varphi},
$$

and that the last term (which we will denote $T_{5}$ ) is equal to

$$
\sum_{j, k=1}^{n} \int_{\partial \Omega} \eta \frac{\partial^{2} r}{\partial z_{j} \partial \bar{z}_{k}} u_{j} \overline{u_{k}} e^{-\varphi} d \sigma
$$

Let again $j, k \in\{1, \ldots, n\}$ be fixed. We transform the single terms that appear in $T_{2}$. In the first step we write

$$
\begin{aligned}
-\int_{\Omega} u_{j} \frac{\partial \eta}{\partial z_{j}} \overline{\delta_{k} u_{k}} e^{-\varphi} d \lambda & \\
& =-2 \operatorname{Re} \int_{\Omega} u_{j} \frac{\partial \eta}{\partial z_{j}} \overline{\delta_{k} u_{k}} e^{-\varphi} d \lambda+\int_{\Omega} \overline{u_{j}} \frac{\partial \eta}{\partial \bar{z}_{j}} \delta_{k} u_{k} e^{-\varphi} d \lambda .
\end{aligned}
$$

The second term on the right side can be computed by the Gauss formula as follows:

$$
\begin{aligned}
\int_{\Omega} \overline{u_{j}} \frac{\partial \eta}{\partial \bar{z}_{j}} \delta_{k} u_{k} e^{-\varphi} d \lambda= & \int_{\Omega} \overline{u_{j}} \frac{\partial \eta}{\partial \bar{z}_{j}} \frac{\partial\left(u_{k} e^{-\varphi}\right)}{\partial z_{k}} d \lambda \\
= & -\int_{\Omega} \frac{\partial^{2} \eta}{\partial \bar{z}_{j} \partial z_{k}} \overline{u_{j}} u_{k} e^{-\varphi} d \lambda-\int_{\Omega} \frac{\partial \overline{u_{j}}}{\partial z_{k}} \frac{\partial \eta}{\partial \bar{z}_{j}} u_{k} e^{-\varphi} d \lambda \\
& +\int_{\partial \Omega} \frac{\partial r}{\partial z_{k}} \frac{\partial \eta}{\partial \bar{z}_{j}} \overline{u_{j}} u_{k} e^{-\varphi} d \sigma
\end{aligned}
$$

We now sum over all $j, k=1, \ldots, n$. Again, the sum of the boundary integrals vanishes because of (10). Likewise, we see that

$$
\sum_{j, k=1}^{n} \int_{\Omega} \frac{\partial \overline{u_{j}}}{\partial z_{k}} \frac{\partial \eta}{\partial \bar{z}_{j}} u_{k} e^{-\varphi} d \lambda=T_{3}
$$

Summation of (13) over all $j, k$ thus yields (by means of (7))

$$
\begin{aligned}
T_{2} & =-\sum_{j, k=1}^{n} \int_{\Omega} u_{j} \frac{\partial \eta}{\partial z_{j}} \overline{\delta_{k} u_{k}} e^{-\varphi} d \lambda \\
& =-2 \sum_{j, k=1}^{n} \operatorname{Re} \int_{\Omega} u_{j} \frac{\partial \eta}{\partial z_{j}} \overline{\delta_{k} u_{k}} e^{-\varphi} d \lambda-\int_{\Omega} \sum_{j, k} \frac{\partial^{2} \eta}{\partial \bar{z}_{j} \partial z_{k}} \overline{u_{j}} u_{k} e^{-\varphi} d \lambda-T_{3} \\
& \left.=2 \operatorname{Re}(u\lrcorner \partial \eta, \bar{\partial}_{\varphi}^{*} u\right)_{\varphi}-\int_{\Omega} \sum_{j, k} \frac{\partial^{2} \eta}{\partial \bar{z}_{j} \partial z_{k}} \overline{u_{j}} u_{k} e^{-\varphi} d \lambda-T_{3} .
\end{aligned}
$$


In order to transform the term $T_{5}$, we need only recall the argument of [Hör, p. 103]. It was shown there that (3) implies

$$
\sum_{k=1}^{n}\left(\frac{\partial \overline{u_{k}}}{\partial z_{j}} \frac{\partial r}{\partial \bar{z}_{k}}+\overline{u_{k}} \frac{\partial^{2} r}{\partial z_{j} \partial \bar{z}_{k}}\right)=0 \quad \text { on } \partial \Omega
$$

for all fixed $j$. We multiply by $u_{j} \eta e^{-\varphi}$ and then sum over all $j$. Finally, integration over $\partial \Omega$ gives us

$$
T_{5}=-\sum_{j, k} \mathcal{I}_{2}(j, k)=\sum_{j, k=1}^{n} \int_{\partial \Omega} \eta \frac{\partial^{2} r}{\partial z_{j} \partial \bar{z}_{k}} u_{j} \overline{u_{k}} e^{-\varphi} d \sigma .
$$

The proof of the a priori formula for $\bar{\partial}$ is complete.

For the proof of (b) we need only observe that, for pseudoconvex $\Omega$, the boundary integral on the right-hand side of (4) is nonnegative if $u \in \mathcal{F}_{1}(\Omega, \varphi)$. Namely, from (10) we see that, for any $\zeta \in \partial \Omega$, the vector $\left(u_{1}(\zeta), \ldots, u_{n}(\zeta)\right)$ belongs to the holomorphic tangent space $T_{\zeta}^{(1,0)} \partial \Omega$. Hence $\mathcal{L}_{r}(\zeta ; u(\zeta)) \geq 0$.

\section{Proof of the Theorem}

We now give the proof of (2) under the assumption that $\psi$ and $V$ are smooth and the codimension of $H$ is $k=1$. (The general case is settled by iterating the result from codimension 1). After a suitable choice of coordinates, we may assume that

$$
H=\left\{z=\left(z^{\prime}, z_{n}\right) \mid z_{n}=0\right\} .
$$

Then $V(z)+2 \log \left|z_{n}\right| \leq C_{V}$ on $D_{t}$. There exists a number $\varepsilon>0$ such that $\left(z^{\prime}, 0\right) \in D^{\prime}$ whenever $z \in D_{t}$ and $\left|z_{n}\right|<\varepsilon$. Let $\chi \in C^{\infty}(\mathbb{R})$ be a function with $\chi(x)=1$ on $(-\infty, 1 / 4]$ and $\chi(x)=0$ on $[3 / 4, \infty)$. For an arbitrary function $f \in$ $H^{2}\left(D^{\prime}, \psi+V\right)$, we define the following $(0,1)$-form:

$$
\alpha_{f}:=\bar{\partial}\left[\chi\left(\frac{\left|z_{n}\right|^{2}}{\varepsilon^{2}}\right) f\left(z^{\prime}, 0\right)\right]=\chi^{\prime}\left(\frac{\left|z_{n}\right|^{2}}{\varepsilon^{2}}\right) f\left(z^{\prime}, 0\right) \frac{z_{n}}{\varepsilon^{2}} d \overline{z_{n}} .
$$

This form is $\bar{\partial}$-closed and smooth on $D_{t}$.

Let

$$
\begin{aligned}
& \mathcal{J}_{\varepsilon}(f) \\
& \quad:=\int_{D^{\prime}}\left|f\left(z^{\prime}, 0\right)\right|^{2} \int_{\left\{1 / 2<\left|z_{n}\right|<1\right\}} \exp \left[-(\psi+V)\left(z^{\prime}, \varepsilon z_{n}\right)\right] d \lambda\left(z_{n}\right) d \lambda\left(z^{\prime}\right) .
\end{aligned}
$$

We look for smooth weight functions $\varphi$ and $\eta>0$ such that $\left(\eta+\eta^{3}\right) e^{-\psi} \leq e^{C_{V}-\varphi}$ and such that the quadratic form $\eta \mathcal{L}_{\varphi}-\mathcal{L}_{\eta}$ tames these data and yields a basic estimate of the form

$$
\begin{aligned}
& \left|\left(u, \alpha_{f}\right)_{\varphi}\right|^{2} \\
& \quad \leq C^{\prime} \mathcal{J}_{\varepsilon}(f)\left\|\sqrt{\eta+\eta^{3}} \bar{\partial}_{\varphi}^{*} u\right\|_{\varphi}^{2} \quad \text { for all }(0,1) \text {-forms } u \in \operatorname{dom}\left(\bar{\partial}_{\varphi}^{*}\right) .
\end{aligned}
$$


For an arbitrary number $0<\tau<\varepsilon^{2}$ we put

$$
\varphi(z)=\varepsilon|z|^{2}+\log \left(\left|z_{n}\right|^{2}+\tau\right)+\psi(z)+V(z)
$$

and

$$
w=V+\log \left(\left|z_{n}\right|^{2}+\varepsilon^{2}\right)-C_{V}-4 .
$$

Then we have $w \leq-3$ on $D_{t}$ if $\varepsilon$ is chosen small enough. Moreover, $w$ is plurisubharmonic. The function

$$
\eta=2(-w+\log (-w))
$$

is plurisuperharmonic, and $-4 w \geq \eta>8$ everywhere. By explicit computation we obtain

$$
-\mathcal{L}_{\eta}=2\left(1-\frac{1}{w}\right) \mathcal{L}_{w}+2 \frac{\partial w \otimes \overline{\partial w}}{w^{2}} \geq 2 \mathcal{L}_{w}+4 \frac{\partial \eta \otimes \overline{\partial \eta}}{\eta^{3}} .
$$

We will prove that (17) is satisfied for these functions $\eta$ and $\varphi$.

It will suffice to check (17) for all $(0,1)$-forms $u \in \mathcal{F}_{t}:=\mathcal{F}_{1}\left(D_{t}, \varphi\right)$, since this space is dense in $\operatorname{dom}\left(\bar{\partial}_{\varphi}^{*}\right)$ with respect to the graph norm

$$
u \mapsto\|u\|_{\varphi}+\|\bar{\partial} u\|_{\varphi}+\left\|\bar{\partial}_{\varphi}^{*} u\right\|_{\varphi} .
$$

(Note that, because of the smoothness assumption on $V$, the function $\gamma:=\sqrt{\eta+\eta^{3}}$ is bounded on each $D_{t}$.) We may furthermore restrict ourselves to forms in the null space $N_{(0,1)}(\bar{\partial})$ of $\bar{\partial}$ (for forms $u \perp N_{(0,1)}(\bar{\partial})$, the estimate (17) is trivial).

Let $u \in \mathcal{F}_{1}\left(D_{t}, \varphi\right) \cap N_{(0,1)}(\bar{\partial})$. We can apply (5). Using (19), we can split the mixed term $\left.(u\lrcorner \partial \eta, \bar{\partial}_{\varphi}^{*} u\right)_{\varphi}$ that appeared in (5). By the Cauchy-Schwarz inequality, we obtain:

$$
\begin{aligned}
\left.-2 \mid(u\lrcorner \partial \eta, \bar{\partial}_{\varphi}^{*} u\right)_{\varphi} \mid & \left.=-2 \mid(u\lrcorner \eta^{-3 / 2} \partial \eta, \eta^{3 / 2} \bar{\partial}_{\varphi}^{*} u\right)_{\varphi} \mid \\
& \geq-\| u\lrcorner \eta^{-3 / 2} \partial \eta\left\|_{\varphi}^{2}-\right\| \eta^{3 / 2} \bar{\partial}_{\varphi}^{*} u \|_{\varphi}^{2} \\
& \geq \frac{1}{4} \int_{D_{t}} \mathcal{L}_{\eta}(z ; u) e^{-\varphi} d \lambda-\left\|\eta^{3 / 2} \bar{\partial}_{\varphi}^{*} u\right\|_{\varphi}^{2} .
\end{aligned}
$$

Let $Q$ denote the quadratic form defined by the coefficients of $\eta \mathcal{L}_{\varphi}-\frac{3}{4} \mathcal{L}_{\eta}$. Substituting (20) into (5), we have

$$
\|\sqrt{\eta} \bar{\partial} u\|_{\varphi}^{2}+\left\|\sqrt{\eta+\eta^{3}} \bar{\partial}_{\varphi}^{*} u\right\|_{\varphi}^{2} \geq \int_{D_{t}} Q(z ; u) e^{-\varphi} d \lambda(z) ;
$$

hence (with $\gamma=\sqrt{\eta+\eta^{3}}$ ),

$$
\int_{D_{t}} Q(z ; u) e^{-\varphi} d \lambda \leq\|\gamma \bar{\partial} u\|_{\varphi}^{2}+\left\|\gamma \bar{\partial}_{\varphi}^{*} u\right\|_{\varphi}^{2} .
$$

The form $Q$ is even positive definite, since

$$
Q \geq \varepsilon \eta \mathcal{L}_{|z|^{2}}+\frac{3}{2} \mathcal{L}_{w} \geq 8 \varepsilon \mathcal{L}_{|z|^{2}}+\frac{\varepsilon^{2}}{\left(\varepsilon^{2}+\left|z_{n}\right|^{2}\right)^{2}} \mathcal{L}_{\left|z_{n}\right|^{2}} .
$$

Thus, $Q^{-1}(z ; u)$ is also meaningful. In combination with the Cauchy-Schwarz inequality, we obtain 


$$
\begin{aligned}
\left|\left(u, \alpha_{f}\right)_{\varphi}\right|^{2} & \leq\left(\int_{D_{t}} Q^{-1}\left(z ; \alpha_{f}\right) e^{-\varphi} d \lambda\right)\left(\int_{D_{t}} Q(z ; u) e^{-\varphi} d \lambda\right) \\
& \leq 2\left(\int_{D_{t}} Q^{-1}\left(z ; \alpha_{f}\right) e^{-\varphi} d \lambda\right)\left(\|\gamma \bar{\partial} u\|_{\varphi}^{2}+\left\|\gamma \bar{\partial}_{\varphi}^{*} u\right\|_{\varphi}^{2}\right)
\end{aligned}
$$

for all $u \in \mathcal{F}_{t}$. On $\operatorname{supp}\left(\alpha_{f}\right)$ we even have

$$
Q \geq 8 \varepsilon \mathcal{L}_{|z|^{2}}+\frac{1}{4 \varepsilon^{2}} d z_{n} \overline{d z_{n}} .
$$

Substituting (15), we derive that

$$
\begin{aligned}
& \int_{D_{t}} Q^{-1}\left(z ; \alpha_{f}\right) e^{-\varphi} d \lambda \\
& \leq C^{\prime} \int_{D_{t} \cap\left\{\varepsilon / 2<\left|z_{n}\right| \leq \varepsilon\right\}}\left|z_{n}\right|^{2} \frac{4}{\varepsilon^{2}}\left|f\left(z^{\prime}, 0\right)\right|^{2} \frac{e^{-\psi-V}}{\left|z_{n}\right|^{2}+\tau} d \lambda \\
& \leq C^{\prime \prime} \varepsilon^{-2} \int_{D^{\prime}}\left|f\left(z^{\prime}, 0\right)\right|^{2} \int_{\left\{\varepsilon / 2<\left|z_{n}\right|<\varepsilon\right\}} e^{-(\psi+V)\left(z^{\prime}, z_{n}\right)} d \lambda\left(z_{n}\right) d \lambda\left(z^{\prime}\right)=C^{\prime \prime} \mathcal{J}_{\varepsilon}(f),
\end{aligned}
$$

where $\mathcal{J}_{\varepsilon}(f)$ is defined as in (16). This yields

$$
\left|\left(u, \alpha_{f}\right)_{\varphi}\right|^{2} \leq C^{*} \mathcal{J}_{\varepsilon}(f)\left\|\gamma \bar{\partial}_{\varphi}^{*} u\right\|_{\varphi}^{2}
$$

for all forms $u \in \mathcal{F}_{t} \cap N_{(0,1)}(\bar{\partial})$ and hence for any $u \in \operatorname{dom}\left(\bar{\partial}_{\varphi}^{*}\right)$. By the HahnBanach theorem combined with the Riesz theorem, we obtain a solution $u_{\varepsilon} \in$ $L^{2}\left(D_{t}, \varphi\right)$ of the equation $\bar{\partial}\left(\gamma u_{\varepsilon}\right)=\alpha_{f}$ such that

$$
\left\|u_{\varepsilon}\right\|_{\varphi}^{2} \leq C^{\prime \prime} \mathcal{J}_{\varepsilon}(f)
$$

The function $u_{\varepsilon}$ is even smooth, and

$$
\tilde{f}_{\varepsilon, \tau}:=\chi\left(\frac{\left|z_{n}\right|^{2}}{\varepsilon^{2}}\right) f\left(z^{\prime}, 0\right)-\gamma u_{\varepsilon}
$$

is holomorphic on $D_{t}$.

We estimate the norm of this function as follows:

$$
\left\|\tilde{f}_{\varepsilon, \tau}\right\|_{\psi}^{2} \leq 2 \int_{D_{t} \cap\left\{\left|z_{n}\right|<\varepsilon\right\}}\left|f\left(z^{\prime}, 0\right)\right|^{2} e^{-\psi} d \lambda+2\left\|\gamma u_{\varepsilon}\right\|_{\psi}^{2}
$$

we have $\left(\varepsilon^{2}+\left|z_{n}\right|^{2}\right)^{-1} e^{-V} e^{C_{V}} \geq 1 / 2$ on $D_{t}$ if $\varepsilon$ is small enough, so

$$
\begin{aligned}
\frac{1}{2} e^{-C_{V}} & \int_{D_{t} \cap\left\{\left|z_{n}\right|<\varepsilon\right\}}\left|f\left(z^{\prime}, 0\right)\right|^{2} e^{-\psi} d \lambda \\
& \leq \int_{D_{t} \cap\left\{\left|z_{n}\right|<\varepsilon\right\}}\left|f\left(z^{\prime}, 0\right)\right|^{2} \frac{1}{\varepsilon^{2}+\left|z_{n}\right|^{2}} e^{-\psi-V} d \lambda \\
& =\int_{D_{t} \cap\left\{\left|z_{n}\right|<1\right\}}\left|f\left(z^{\prime}, 0\right)\right|^{2} \frac{1}{1+\left|z_{n}\right|^{2}} e^{-(\psi+V)\left(z^{\prime}, \varepsilon z_{n}\right)} d \lambda \\
& \leq \mathcal{J}_{\varepsilon}^{*}(f):=\int_{\left\{\left|z_{n}\right|<1\right\}}\left|f\left(z^{\prime}, 0\right)\right|^{2} e^{-(\psi+V)\left(z^{\prime}, \varepsilon z_{n}\right)} d^{2 n} z .
\end{aligned}
$$


Furthermore, since for $\tau<\varepsilon^{2}$ we can estimate

$$
V(z)+\log \left(\left|z_{n}\right|^{2}+\tau\right) \leq w(z)+C_{V}+4 \leq-\frac{\eta(z)}{4}+4+C_{V},
$$

the second term in (24) is dominated by some constant times $e^{C_{V}} \mathcal{J}_{\varepsilon}(f)$, because

$$
\begin{aligned}
\left|\gamma u_{\varepsilon}\right|^{2} e^{-\psi} & =\left(\eta+\eta^{3}\right) e^{\varepsilon|z|^{2}+V+\log \left(\left|z_{n}\right|^{2}+\tau\right)}\left|u_{\varepsilon}\right|^{2} e^{-\varphi} \\
& \leq C^{\prime} C_{t}^{\varepsilon} e^{C_{V}}\left(\eta+\eta^{3}\right) e^{-\eta / 4}\left|u_{\varepsilon}\right|^{2} e^{-\varphi} \leq C^{\prime \prime} C_{t}^{\varepsilon} e^{C_{V}}\left|u_{\varepsilon}\right|^{2} e^{-\varphi},
\end{aligned}
$$

where $C_{t}=\exp \left(\max _{D_{t}}|z|^{2}\right)$ and $C^{\prime}, C^{\prime \prime}>0$ are unimportant constants.

We next show that, for any point $\left(z^{\prime}, 0\right) \in D_{t} \cap H$, there exists a constant $C=$ $C^{\prime}\left(\varepsilon, z^{\prime}, t\right)$ such that, for all $0<\tau<\varepsilon^{2}$,

$$
\left|\tilde{f}_{\varepsilon, \tau}\left(z^{\prime}, 0\right)-f\left(z^{\prime}, 0\right)\right|^{2}=\left|\gamma u_{\varepsilon}\left(z^{\prime}, 0\right)\right|^{2} \leq C\left(z^{\prime}, \varepsilon, t\right) \frac{1}{\log \left(1+\varepsilon^{2} / 4 \tau\right)}\left\|u_{\varepsilon}\right\|_{\varphi}^{2} .
$$

Here we have used the fact that $\gamma u_{\varepsilon}$ is holomorphic on $\left\{\left|z_{n}\right|<\varepsilon / 2\right\}$. It satisfies on $D_{t}$ the upper estimate

$$
\frac{\left|\gamma u_{\varepsilon}\right|^{2}}{\left|z_{n}\right|^{2}+\tau}=\gamma^{2} e^{\varepsilon|z|^{2}+\psi+V}\left|u_{\varepsilon}\right|^{2} e^{-\varphi} \leq C(t, \varepsilon)\left|u_{\varepsilon}\right|^{2} e^{-\varphi},
$$

with a constant $C(t, \varepsilon)$ that does not depend on $\tau$. Let $\left(z^{\prime}, 0\right) \in D_{t} \cap H$ be arbitrary. Then, after shrinking $\varepsilon$ if necessary, we find a radius $\rho\left(z^{\prime}\right)$ such that the polydisc $P\left(z^{\prime}\right)=\Delta_{n-1}\left(z^{\prime}, \rho\left(z^{\prime}\right)\right) \times \Delta(0, \varepsilon / 2)$ is contained in $D_{t}$. Let

$$
\gamma u_{\varepsilon}(w)=\sum_{\beta \in \mathbb{N}_{0}^{n}} A_{\beta}\left(z^{\prime}\right)\left(w-\left(z^{\prime}, 0\right)\right)^{\beta}
$$

denote the Taylor expansion of $\gamma u_{\varepsilon}$ about $\left(z^{\prime}, 0\right)$ on $P\left(z^{\prime}\right)$. Then, using the orthogonality of the monomials $\left(w-\left(z^{\prime}, 0\right)\right)^{\beta}$ in conjunction with (26), we have

$$
\left|\gamma u_{\varepsilon}\left(z^{\prime}, 0\right)\right|^{2} \int_{P\left(z^{\prime}\right)} \frac{1}{\left|w_{n}\right|^{2}+\tau} d \lambda(w) \leq \int_{P\left(z^{\prime}\right)} \frac{\left|\gamma u_{\varepsilon}(w)\right|^{2}}{\left|w_{n}\right|^{2}+\tau} d \lambda(w) \leq C(t, \varepsilon)\left\|u_{\varepsilon}\right\|_{\varphi}^{2} .
$$

From

$$
\int_{P\left(z^{\prime}\right)} \frac{1}{\left|w_{n}\right|^{2}+\tau} d \lambda(w)=C_{n}^{*} \rho\left(z^{\prime}\right)^{2 n-2} \log \left(1+\frac{\varepsilon^{2}}{4 \tau}\right),
$$

with an unimportant constant $C_{n}^{*}$ we obtain (25). We saw already that $\left\|\tilde{f}_{\varepsilon, \tau}\right\|_{\psi}^{2} \leq$ $C^{\prime} C_{t}^{\varepsilon} e^{C_{V}}\left(\mathcal{J}_{\varepsilon}(f)+\mathcal{J}_{\varepsilon}^{*}(f)\right)$ for all $\tau<\varepsilon^{2}$, with a constant $C_{t}$ that does not depend on anything but $t$. After selecting a weak- $\star$-convergent subsequence $\left(\tilde{f}_{\varepsilon, \tau_{j}}\right)_{j}$ from the $\tilde{f}_{\varepsilon, \tau}$, we obtain by means of $(25)$ an extension $\tilde{f}_{\varepsilon} \in H^{2}\left(D_{t}, \psi\right)$ of $f$. Finally we let $\varepsilon$ tend to zero. Then $\mathcal{J}_{\varepsilon}(f) \rightarrow 3 \pi / 4\|f\|_{D^{\prime}, \psi+V}^{2}$ and $\mathcal{J}_{\varepsilon}^{*}(f) \rightarrow \pi\|f\|_{D^{\prime}, \psi+V}^{2}$. After once more choosing a weak- $\star$-convergent subsequence from the $\tilde{f}_{\varepsilon}$, we will gain the desired extension $\tilde{f}_{t} \in H^{2}\left(D_{t}, \psi\right)$, satisfying (2).

By checking all the steps in the foregoing proof, we see that we indeed obtain our next result, which generalizes the case of codimension 1 . 
3.2. THEOREM. Let $D$ be a pseudoconvex domain in $\mathbb{C}^{n}$ and $h$ a holomorphic function such that $Z_{h}=\{h=0\}$ becomes a 1-codimensional complex submanifold of $D$. Assume that a plurisubharmonic function $V$ exists on $D$ for which

$$
C_{V, h}=\sup _{D}(V+2 \log |h|)<\infty .
$$

Then there exists a constant $C_{n}>0$ such that, for all plurisubharmonic functions $\psi$ on $D$, one can find a bounded linear extension operator $E_{\psi}^{V, h}: H^{2}\left(D \cap Z_{h}\right.$, $\psi+V) \rightarrow H^{2}(D, \psi)$ whose operator norm can be estimated by $\left\|E_{\psi}^{V, h}\right\|^{2} \leq$ $C_{n} e^{C_{V, h}}$, with a constant $C_{n}$ that does not depend on anything but the dimension.

\section{An Application to the Bergman Kernel}

We want to give an application of the Ohsawa extension theorem to the Bergman kernel of a class of pseudoconvex domains with a $C^{2}$-smooth boundary-an application covering all domains that are regular in the sense of [DF].

Let $\Omega \subset \subset \mathbb{C}^{n}$ be a pseudoconvex domain. For a plurisubharmonic function $u$ on $\Omega$, we denote by $K_{\Omega, u}$ the Bergman kernel for the Hilbert space $H^{2}(\Omega, u)$ of all holomorphic functions $f$ such that $\int_{\Omega}|f|^{2} e^{-u} d \lambda<\infty$. As usual we put $K_{\Omega}=$ $K_{\Omega, 0}$. Furthermore, we define $\mathcal{P}(\bar{\Omega})$ as the family of all functions that are continuous on $\bar{\Omega}$ and plurisubharmonic on $\Omega$. Let $\delta_{\Omega}$ denote the boundary distance function on $\Omega$.

If now $H$ is an affine linear subspace of codimension $k$ that meets $\Omega$, and if $u$ is a plurisubharmonic function on $\Omega$ satisfying

$$
u(z)+2 k \log \operatorname{dist}(z, D \cap H) \leq 0
$$

on $\Omega$, then from Theorem 0.1 we obtain, for all $w \in \Omega \cap H$,

$$
K_{\Omega}(w) \geq C_{n} K_{\Omega \cap H, u}(w) .
$$

Our result on the Bergman kernel is as follows.

4.1. THEOREM. Assume that $\partial \Omega \in C^{2}$ and that each point $\zeta \in \partial \Omega$ is a peak point for $\mathcal{P}(\bar{\Omega})$. Let $z^{0} \in \partial \Omega$ be a point such that the Levi form of $\partial \Omega$ has $p<n-1$ positive eigenvalues at $z^{0}$. Then, for the Bergman kernel of $\Omega$, we have

$$
\lim _{\Omega \ni w \rightarrow z^{0}} \delta_{\Omega}(w)^{p+2} K_{\Omega}(w)=\infty .
$$

REMARKS. (a) In (28), the approach of $w \in \Omega$ toward $z^{0}$ is not required to be nontangential.

(b) By work of Sibony [Si], it is known that the hypothesis concerning the plurisubharmonic peak functions is satisfied when $\Omega$ is regular in the sense of [DF].

(c) Stronger quantitative estimates have been obtained for the large class of domains of finite type. See, for example, [DHOh] or [Cat] and the references given in those papers. 
(d) An extremely large Levi degeneracy set $E$ of the boundary is not an obstacle for (28) to hold. One should note that Sibony [Si] found pseudoconvex regular domains in $\mathbb{C}^{2}$ such that $E$ has a positive Hausdorff measure of dimension 3.

Before giving a proof of the theorem, we summarize some facts about plurisubharmonic peak functions. In [Si, Thm. 2.1], the following is proved.

4.2. Lemma. Assume that $G \subset \subset \mathbb{C}^{n}$ is pseudoconvex, with a $C^{1}$-smooth boundary, such that each point $\zeta \in \partial G$ is a peak point for $\mathcal{P}(\bar{G})$. If $u \in C^{0}(\partial G)$, then the function

$$
\tilde{u}(z):=\sup \{v(z) \mid v \in \mathcal{P}(\bar{G}), v \leq u \text { on } \partial G\}
$$

is also an element of $\mathcal{P}(\bar{G})$; moreover, $\tilde{u} \mid \partial G=u$.

Let now $G$ be as in the lemma, and let $d_{q}(z):=|z-q|^{2}$ for $q \in \partial G$ and $z \in \mathbb{C}^{n}$. Then we have our next lemma.

4.3. LEMma. The functions $\psi_{q}:=\widetilde{-d}_{q}$, where $q \in \partial G$, have the following properties:

(a) $\psi_{q} \leq-d_{q}$ on $\bar{G}$;

(b) $\left|\psi_{p}-\psi_{q}\right| \leq 2 \operatorname{diam}(G) \cdot|p-q|$ for $p, q \in \partial G$.

Proof. (a) is a consequence of the maximum principle. Let us prove (b). For $\zeta \in$ $\partial G$, we have

$$
\begin{aligned}
\psi_{q}(\zeta)+2 \operatorname{Re}\langle\zeta-p, p-q\rangle & =-|\zeta-q|^{2}+2 \operatorname{Re}\langle\zeta-p, p-q\rangle \\
& =-|\zeta-p|^{2}-|p-q|^{2} \leq-d_{p}(\zeta)
\end{aligned}
$$

Hence $z \mapsto \psi_{q}(z)+2 \operatorname{Re}\langle z-p, p-q\rangle$ is a candidate for the supremum that defines $\psi_{p}$, and therefore

$$
\psi_{q}(z) \leq \psi_{p}(z)+2|\operatorname{Re}\langle z-p, p-q\rangle| \leq \psi_{p}(z)+2 \operatorname{diam}(G) \cdot|p-q| .
$$

Since the roles of $p$ and $q$ can be interchanged, the claim now follows.

Proof of Theorem 4.1. Choose open neighborhoods $W \subset \subset V$ for $z^{0}$, a smoothly bounded pseudoconvex domain $D \subset \subset \mathbb{C}^{n}$, and a linear subspace $E$ of $\mathbb{C}^{n}$ of dimension $p+1$ such that the following hold.

(i) $D \cap V \subset \Omega \cap V$ and $\partial D \cap V=\partial \Omega \cap V$.

(ii) For each $\zeta \in \partial \Omega \cap V$, the intersection $D_{\zeta}^{\prime}:=(\zeta+E) \cap D$ is strongly pseudoconvex and has a $C^{2}$-smooth boundary.

(iii) There is a number $\lambda>0$ such that the eigenvalues of the Levi form of $\partial D_{\zeta}^{\prime}$ are bounded from below by $\lambda$ whenever $\zeta \in \partial \Omega \cap V$.

(iv) For any point $w \in \Omega \cap W$, there exists $w^{*} \in \partial \Omega \cap V$ with $w \in D_{w^{*}}^{\prime}$ and $\left|w-w^{*}\right| \approx \delta_{\Omega}(w)$.

By the localization lemma for the Bergman kernel (see e.g. [Oh1]) we have, with a constant $C$ that depends only on $V$ and $W$,

$$
K_{\Omega} \geq C K_{\Omega \cap V}=C K_{D \cap V} \geq C K_{D}
$$


on $\Omega \cap W$. Let $w \in \Omega \cap W$ be an arbitrary point, and let $w^{*}$ be a boundary point according to (iv). Then, using Lemma 4.3(a) we can apply formula (27) to the function

$$
u:=-(n-p-1) \log \left(-\psi_{w^{*}}\right)
$$

and obtain (with a new constant $C^{\prime}$ )

$$
K_{D}(w) \geq C^{\prime} K_{D_{w^{*}, u}^{\prime}}(w) .
$$

But in $[\mathrm{DHM}]$ it is shown that (with a constant that depends only on $\lambda$ ):

$$
K_{D_{w^{*}, u}^{\prime}}(w) \geq C_{\lambda}\left|w-w^{*}\right|^{-p-2} e^{u(w)} \geq C^{\prime \prime} \delta_{\Omega}(w)^{-p-2}\left|\psi_{w^{*}}(w)\right|^{-(n-p-1)} .
$$

Lemma 4.3(b) implies that, for all $w \in \Omega \cap W$,

$$
\left|\psi_{w^{*}}(w)\right| \leq\left|\psi_{z^{0}}(w)\right|+\left|w^{*}-z^{0}\right| \leq \hat{C}\left(\left|\psi_{z^{0}}(w)\right|+\delta_{\Omega}(w)+\left|w-z^{0}\right|\right)
$$

and, consequently,

$$
\delta_{\Omega}(w)^{p+2} K_{\Omega}(w) \geq \hat{C} \frac{1}{\left(\left|\psi_{z^{0}}(w)\right|+\delta_{\Omega}(w)+\left|w-z^{0}\right|\right)^{n-p-1}} .
$$

From this the theorem follows.

\section{References}

[BoS] J. Boas and E. J. Straube, Global regularity of the $\bar{\partial}$-Neumann problem: A survey of the $L^{2}$-Sobolev theory, Current developments in several complex variables (M. Schneider, Y. T. Siu, eds.), Proc. of the special year in several complex variables at the MSRI, Cambridge Univ. Press (forthcoming).

[BonD] P. Bonneau and K. Diederich, Integral solution operators for the CauchyRiemann equations on pseudoconvex domains, Math. Ann. 286 (1990), 77-100.

[Bou] T. Bouche, Inegalités de Morse pour la d"-cohomologie sur une variété holomorphe non-compacte, Ann. Sci. École Norm. Sup. (4) 22 (1989), 501-513.

[Bs] B. Berndtsson, The extension theorem of Ohsawa-Takegoshi and the theorem of Donnelly-Fefferman, Ann. Inst. Fourier (Grenoble) 14 (1996), 1083-1094.

[Cat] D. W. Catlin, Estimates of invariant metrics on pseudoconvex domains of dimension two, Math. Z. 200 (1989), 429-466.

[De] J. P. Demailly, Estimations $L^{2}$ pour l'opérateur $\bar{\partial}$ d'un fibré vectoriel holomorphe semi-positif au-dessus d'une variété kählérienne complète, Ann. Sci. École Norm. Sup. (4) 15 (1982), 457-511.

[DF] K. Diederich and J. E. Fornaess, Pseudoconvex domains: Existence of Stein neighborhoods, Duke Math. J. 44 (1977), 641-662.

[DH1] K. Diederich and G. Herbort, Extension of holomorphic $L^{2}$-functions with weighted growth conditions, Nagoya Math. J. 126 (1992), 141-157.

[DH2] - Geometric and analytic boundary invariants on pseudoconvex domains: Comparison results, J. Geom. Anal. 3 (1993), 237-267.

[DHM] K. Diederich, G. Herbort, and V. Michel, Weights of holomorphic extension and restriction, J. Math. Pures Appl. (9) 77 (1998), 697-719.

[DHOh] K. Diederich, G. Herbort, and T. Ohsawa, The Bergman kernel on uniformly extendable pseudoconvex domains, Math. Ann. 273 (1986), 471-478. 
[Hör] L. Hörmander, $L^{2}$-estimates and existence theorems for the $\bar{\partial}$ operator, Acta Math. 113 (1965), 89-152.

[JP] M. Jarnicki and P. Pflug, Bergman completeness of complete circular domains, Ann. Polon. Math. 50 (1989), 219-222.

[McN] J. McNeal, On large values of $L^{2}$-holomorphic functions, Math. Res. Lett. 3 (1996), 247-259.

[Mv] L. Manivel, Un théorème de prolongement $L^{2}$ de sections holomorphes d'un fibré hermitien, Math. Z. 212 (1993), 107-122.

[Oh1] T. Ohsawa, Boundary behavior of the Bergman kernel function on pseudoconvex domains, Publ. Res. Inst. Math. Sci. 20 (1984), 897-902.

[Oh2] - On the extension of $L^{2}$ holomorphic functions II, Publ. Res. Inst. Math. Sci. 24 (1988), 265-275.

[Oh3] - On the extension of $L^{2}$ holomorphic functions III: Negligible weights, Math. Z. 219 (1995), 215-225.

[OhT] T. Ohsawa and K. Takegoshi, On the extension of $L^{2}$-holomorphic functions, Math. Z. 195 (1987), 197-204.

[Si] N. Sibony, Une classe des domaines pseudoconvexes, Duke Math. J. 55 (1987), 299-319.

[Siu] Y. T. Siu, The Fujita conjecture and the extension theorem of OhsawaTakegoshi, Geometric complex analysis (Junjiro Noguchi et al., eds.), pp. 577592, World Scientific, Rivers Edge, NJ, 1996.

K. Diederich

Bergische Unversitaet-

Gesamthochschule Wuppertal

Fachbereich Mathematik

Gauss-Strasse 20

D-42097 Wuppertal

Germany

klas@math.uni-wuppertal.de
G. Herbort

Bergische Unversitaet-

Gesamthochschule Wuppertal

Fachbereich Mathematik

Gauss-Strasse 20

D-42097 Wuppertal

Germany

gregor@math.uni-wuppertal.de 